\title{
Angular Backscatter Filtering with an Immersion Lens SEM
}

\author{
B. Lich ${ }^{* *}$, L. Novak ${ }^{*}$, E. Bosch*, D.J. Stokes ${ }^{* *}$, D.W. Phifer ${ }^{* *}$ and L. Tuma* \\ * FEI Company, Podnikatelska 6, 61200 Brno, Czech Republic \\ *FEI Company, Building AAE, PO Box 80066, 5600 KA Eindhoven, The Netherlands
}

Over the last few decades the use of scanning electron microscopy (SEM) in the characterization of materials has shifted from the simple imaging case to a more complex, multi-modal imaging and analysis model, using the various signals that are available as a result of beam-specimen interactions. These signals can be collected simultaneously and even mixed in real time, to enrich the quality and ease-of-interpretation of data.

The value of using secondary electron (SE) imaging information in addition to backscattered electron (BSE) imaging, as well as the analytical possibilities such as energy dispersive spectroscopy (EDS), electron backscattered electron diffraction (EBSD) and wavelength dispersive spectroscopy (WDS) is widely recognised, but of growing importance is the ability to also differentiate different energy bands in SE [1] and BSE signals [2], allowing us to extract even more knowledge from the SEM image.

The aim of the current work is thus to show the difference in image information that can be selectively obtained at different solid angles of BSE signal. This idea is illustrated schematically in Fig. 1. We demonstrate that various contrast mechanisms can be utilised by employing a segmented detector in combination with microscope parameters such as tilt angle and specimen bias.

Further we demonstrate how the detection efficiency of various angles of the BSE signal can be optimized using a magnetic immersion field in combination with stage bias. Experimental results can also be compared with simulations, to yield a greater understanding of the original of signals and their correlation with specific features of the specimen.

In Fig 1 we show the schematic of the sample with the segmented backscatter detector with an indication in which segment the backscatters were detected as a function of their exit angle from the specimen in a specific setting of the microscope. We will compare these experimental results with the Monte Carlo simulations of the electron paths in an immersion field system and demonstrate that using various microscope parameters like immersion field strength, specimen bias and specimen tilt yield practical usable applications, for instance for grain orientation studies.

\section{References}

[1] P. Kazemian et al, Ultramicroscopy 107, 2007, 140-150

[2] P Hennig et al, J Applied Physics 102(1), 2007

[3] I. Mullerova et al, Materials Transactions, Vol. 48, No. 5, 2007, pp. 1-4 

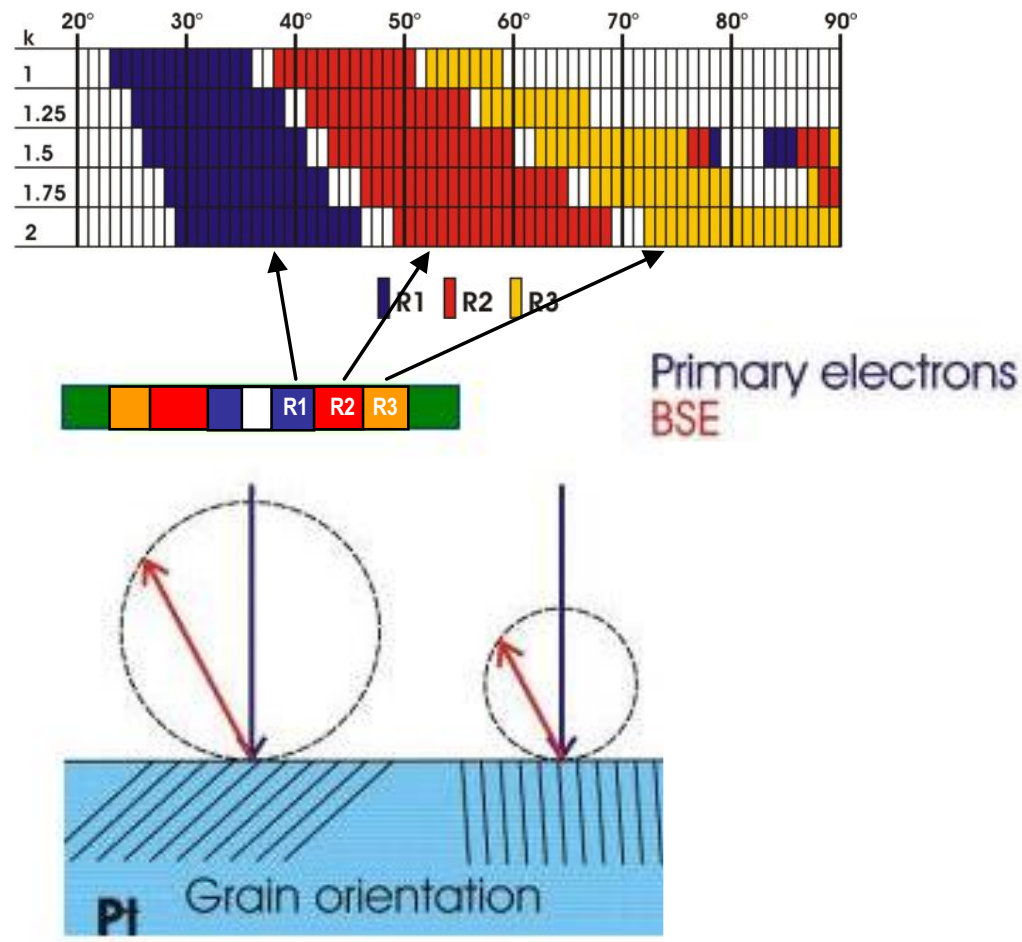

Figure 1 Schematic diagram to BSE detector with high and low angle segments
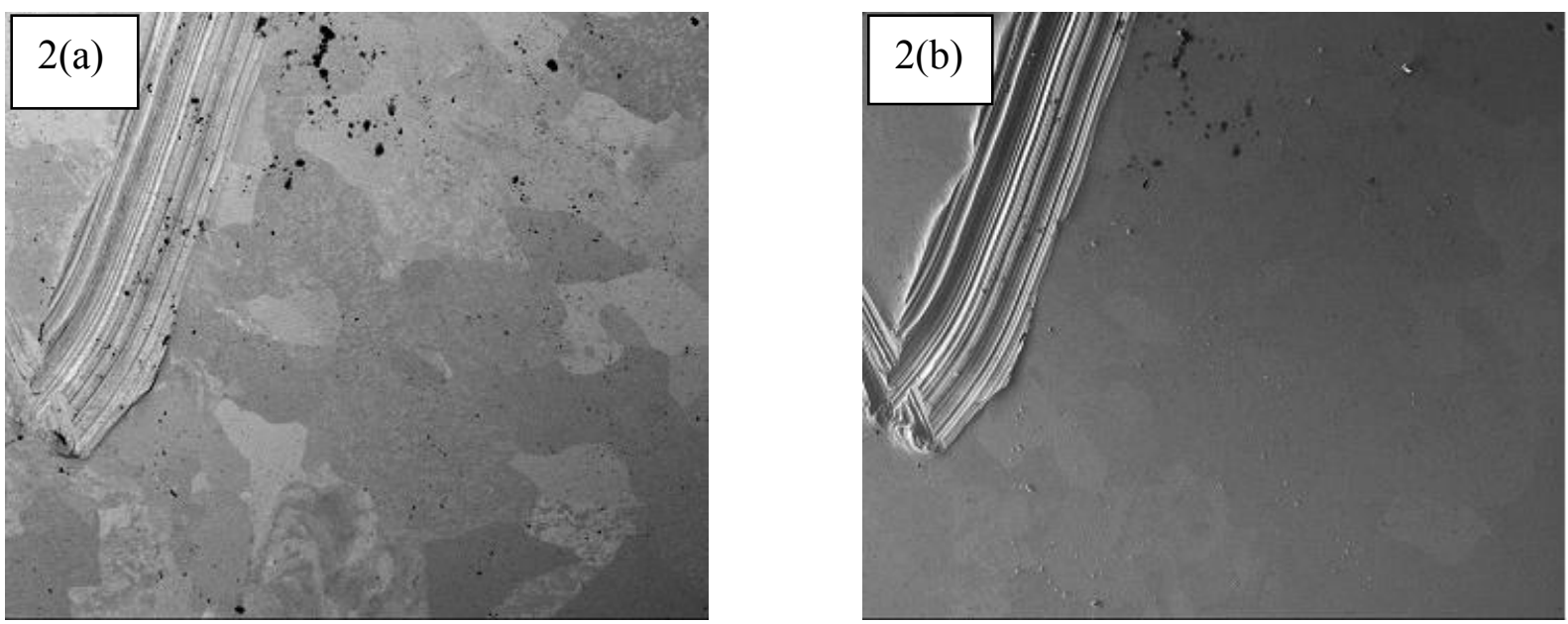

Figure 2 (a) BSE image of Pt sample showing the effects high angle BSE collection. Note the enhanced orientation contrast from the polycrystalline grains. (b) BSE image of the same region as in (a), but using low angle backscatters. Grain contrast is much reduced. 